\title{
A Robust Detector Using SNR with Adaptive Threshold Scheme in Cognitive Radio Networks
}

\author{
ASHISH BAGWARI ${ }^{1}$, JYOTSHANA KANTI ${ }^{2}$, GEETAM SINGH TOMAR $^{3}$, and \\ ASHRAF SAMARA ${ }^{4}$ \\ ${ }^{1,2}$ Uttarakhand Technical University, Dehradun, INDIA, \\ ${ }^{3}$ Machine Intelligence Research Labs, Gwalior, INDIA and THDC-IHET Tehri \\ ${ }^{4}$ Department of Electrical Engineering \\ Iashishbagwari@ieee.org, ${ }^{2}$ jyotshanakanti@gmail.com, ${ }^{3}$ gstomar@ieee.org, \\ ${ }^{4}$ samara_ashraf@yahoo.com
}

\begin{abstract}
Spectrum sensing is a key functionalities of Cognitive radio networks (CRN). There are several methods to improve sensing techniques. In this paper, we present a robust detector using signal to noise ratio (SNR) with adaptive threshold (ADT) scheme in Cognitive Radio Networks (CRN). This technique has two detectors, energy detector (ED)\&EDwithADT detector, out of these two detectors only one will perform sensing operation at a time. Selection of detector depends on condition between estimated SNR value $\left(S_{e}\right)$ and threshold $(\gamma)$. Numerical results show that proposed ESNR_ADT scheme optimizes detection performance and outperforms the cyclostationary based sensing method and adaptive spectrum sensing (SS) by $30.5 \%$ and $30 \%$ at - $10 \mathrm{~dB}$ signal to noise ratio (SNR) respectively. It is also shown that the proposed scheme yields lesser sensing time than cyclostationary detection and adaptive SS scheme in the order of $5.2 \mathrm{~ms}$ and $1.0 \mathrm{~ms}$ at $-20 \mathrm{~dB}$ SNR respectively.
\end{abstract}

Keywords- Cognitive Radio Networks, Estimated signal to noise ratio, Energy detector, Spectrum sensing, Adaptive threshold

\section{Introduction}

Cognitive Radio Network (CRN) is a future based technology works over Dynamic spectrum allocation (DSA) scheme. CRN has four basic functions such as spectrum sensing, spectrum sharing, spectrum mobility, and spectrum decision. In which spectrum sensing technique makes an important role to detect primary user (PU) signal. CRN comes under IEEE 802.22 wireless regional area networks (WRAN) standard, has capability to sense channel usage. It consists primary user (PU) which is licensed user, and secondary user (SU) or CR which is unlicensed user. CRN utilizes PU frequency band between CR users for communication when the licensed band is not used by PU. Detecting PU signal by CR's there are three basics detection methods, named as Matched filter detection method, Energy detection method, and Cyclostationary feature detection method [1-5]. Time-to-time there was various methods have been proposed to improve these SS techniques. Sensing a wide-band spectrum makes an important role in CR. But a few works have been done by some researchers on the wide-band spectrum sensing field in CRs. Two-stage spectrum detecting method is considered as one of the methods to handle this issue. In [6], authors presented a two-stage sensing scheme where first stage consists coarse sensing based on energy detection and, fine sensing based on cyclostationary detection exists in the second stage for SS. If require, coarse stage detects PU signal. Otherwise, fine sensing stage will detect and give the final decision. But the difficulty with 
this scheme is that both stages perform SS, therefore, it increases spectrum sensing time. In [7], author presented another scheme, where out of two stages only one of the detection techniques was running at a time based on the estimated SNR [8]. Although this scheme reduces the mean sensing time but it does not consider spectrum sensing failure problem [9].

In this paper, we have focused and mitigated sensing failure problem [9] by presented a novel collaborative spectrum sensing technique based on estimated SNR with adaptive threshold (ESNR_ADT) in CRN. The propose scheme estimates SNR and based on this selects either ED with fixed threshold or ED with adaptive threshold, to improves the detection performance at a fixed probability of false alarm $\left(P_{f}\right)$ i.e. 0.1. Selection of detector depends on estimated SNR value $\left(S_{e}\right)$ and threshold $(\gamma)$, if $S_{e}$ is greater or equal to $\gamma$ then ED with fixed threshold $\left(\lambda_{l}\right)$ will be selected, else ED with ADT will performsensing operations. ED with ADT uses adaptive threshold which is made adaptive to the fluctuation of the received signal power in each local detector of CRuser. Performance of detector was also calculated in terms of sensing time. It is shown that the proposed sensing technique has smaller SS time than cyclostationary detection and adaptive SS.

The rest of the paper is organized as follows: Section II presents system description. Section III describes proposed system model. Section IV shows the numerical results and analysis. Finally, Section V concludes the paper.

\section{System Description}

In this method, there is a mathematical expression to detect the PU signal by using following hypothesis for received signal $[1,10]$

$$
x(n)= \begin{cases}w(n), & H_{0} \\ s(n) h(n)+w(n), & H_{1}\end{cases}
$$

$x(n)$ shows signal received by each CR user. $s(n)$ is the PU licensed signal, $w(n)$ shows AWGN (additive white gaussian noise) with zero mean i.e. $w(n) \sim \square\left(0, \sigma_{w}{ }^{2}\right)$ and $\sigma_{w}{ }^{2}$ is noise variance, $h(n)$ is the gain of Rayleigh fading channel where wireless channel is Rayleigh exist between the PU and the CR users. $H_{0}$ is the null hypothesis, shows the absence of $\mathrm{PU}$ and $H_{l}$ is the alternative hypothesis, shows that $\mathrm{PU}$ is present.

\section{A. Estimated Signal to Noise Ratio}

Signal to noise ratio (SNR) shows the ratio between signal power (PS) and noise power $(P W)$. Signal power is, received PU signal by CRand noise signal is unwanted signal. We can calculate the power of any kind of signal either PU signal or noise signal.

There is a mathematical formula to calculate estimated $\operatorname{SNR}\left(S_{e}^{\prime}\right)$ value given as

$$
\begin{aligned}
& S_{e}^{\prime}=\frac{P_{s}}{P_{w}} \\
& S_{e}^{\prime}=\frac{\frac{1}{N} \sum_{n=1}^{N}|x(n)|^{2}}{\frac{1}{N} \sum_{n=1}^{N}|w(n)|^{2}}
\end{aligned}
$$

Equation (3) shows estimated SNR value. Where $N$ is total number of received samples, $n=1,2,3 \ldots \ldots \ldots . N$. Once the value of $S_{e}^{\prime}$ is calculated then converts $S_{e}^{\prime}$ into dB, compare $S_{e}$ to decided threshold $(\gamma)$ as shown below in Equation (4), (5) \& (7) respectively. Based on the final resultant of Equation (7) detector will choose any one of the spectrum detector between ED and ED with ADT detectors. 
$S_{e}^{\prime}=\frac{\frac{1}{N} \sum_{n=1}^{N}|x(n)|^{2}}{\frac{1}{N} \sum_{n=1}^{N}|w(n)|^{2}}$

$S_{e \mid d B}=10 \times \log _{10} S_{e}^{\prime}$

$S_{e \mid d B}=10 \times \log _{10}\left[\frac{\frac{1}{N} \sum_{n=1}^{N}|x(n)|^{2}}{\frac{1}{N} \sum_{n=1}^{N}|w(n)|^{2}}\right]$

$S_{e \mid d B} \geq \gamma$

Using Equation (7), if estimated SNR value in $\mathrm{dB}\left(S_{e}\right)$ is greater than or equal to threshold $(\gamma)$ then propose system will select ED to detect PU signal, else ED with ADT will be selected to perform SS operation.

\section{B. Adaptive Threshold Scheme for Spectrum Sensing (ADT)}

ED based SS [11], noise uncertainty arises the difficulty in setting the ideal threshold for a CR and therefore reduces its spectrum sensing reliability [12], Moreover this may not be optimum under low SNRs where the performance of fixedthreshold $\left(\lambda_{1}\right)$ based ED can fluctuate from the desired targeted performance metrics significantly.

In Figure 1, x-axis shows the power level of signals and $y$-axis shows the signals probability. There are two curves, depicts the PU signal and noise curve. According to CRN scheme, it is very easy to detect PU and noise if both are separate from each other. Like ED receives PU signal then it shows $H_{l}$ i.e. channel is occupied, and if receives noise signal it shows $H_{0}$ i.e. channel is un-occupied. But if PU signal and noise both intersects to each other then it is very difficult to sense desired signals. In Figure 1, the area comes between PU and noise curve or under upper bound $\left(\lambda_{A 1}\right)$ and lower bound $\left(\lambda_{A 2}\right)$ is known as confused region. In this region using single threshold detection of noise and PU signal is difficult. In the ED with ADT scheme, the upper bound threshold $\left(\lambda_{A l}\right)$ is selected according to the maximum noise variance, and the lower bound threshold $\left(\lambda_{A 2}\right)$ is selected according to the minimum noise variance.

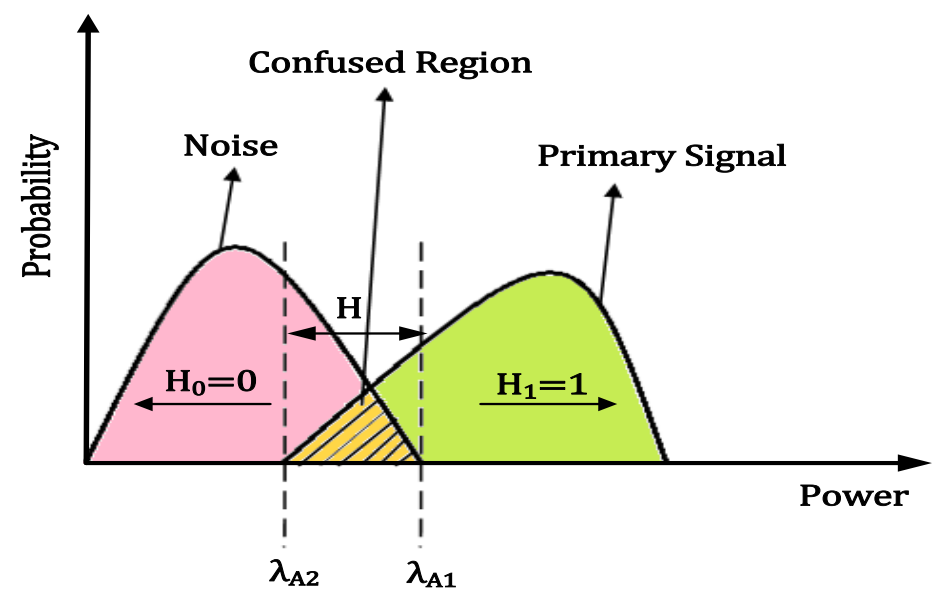

Figure 1. Energy Distribution Curve of PU Signal and Noise 
Figure 1, illustrates the energy distribution curve of PU signal and noise. Using single threshold, detection between noise and PU signal is difficult under confused region. To overcome this problem we considered adaptive two threshold scheme to define the local decision at the $\mathrm{CR}$ user as following logic function rule $(L R)$.

$L R=\left\{\begin{array}{lr}H_{0}=0, & Z \leq \lambda_{A 2} \\ H=M, & \lambda_{A 2}<Z<\lambda_{A 1} \\ H_{1}=1, & \lambda_{A 1} \leq Z\end{array}\right.$

$M$ is the quantization decision, and $Z$ denotes received signal energy by CR user.

In [13], authors proposed a double thresholds method to reduce the average number of sensing bits to the receiver. But, the decreases are achieved at the expense of some sensing performance loss which is sensing failure problem. To remove these problems effectively, two thresholds $\lambda_{A l}$ and $\lambda_{A 2}$ are used to help the decision of the CR user, as shown in Figure 2. Here, two-bit quantization method divides confused region into four equal quantization intervals as $\left(\lambda_{A 2} \mathrm{~A}-\mathrm{AB}-\mathrm{BC}-\mathrm{C} \lambda_{A l}\right)$, where $\lambda_{A 2}, \mathrm{~A}, \mathrm{~B}, \mathrm{C}, \& \lambda_{A l}$ are sub-thresholds $(S T)$ and their values are chosen as

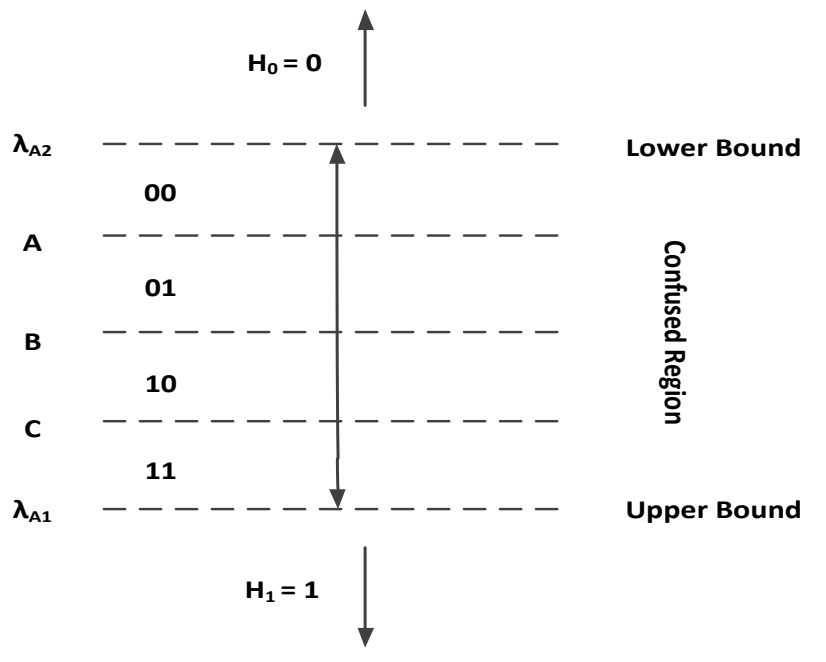

Figure 2. Confused Region is Divided Into Four Equal Levels Using Two-Bit Quantization Method

$$
\begin{aligned}
& S T=\left\{\begin{array}{l}
A=\lambda_{A 2}+D \\
B=A+D \\
C=B+D \\
\lambda_{A 1}=C+D
\end{array}\right. \\
& D=\frac{(\text { Uppere bound }- \text { Lower bound })}{\text { No. of Quantization intervals }}=\frac{\left(\lambda_{A 1}-\lambda_{A 2}\right)}{4} \\
& M= \begin{cases}00, & \lambda_{A 2}<X \leq A \\
01, & A<X \leq B \\
10, & B<X \leq C \\
11, & C<X<\lambda_{A 1}\end{cases}
\end{aligned}
$$


Now, the false alarm probability $(P f)$ of conventional ED with single threshold can be calculated as [12]

$P_{f}=Q\left(\frac{\lambda-N \sigma_{\omega}^{2}}{\sqrt{2 N \sigma_{\omega}^{4}}}\right)$

Where $Q()$ is known as Gaussian tail probability $Q$-function, and noise variance is denoted by $\sigma_{\omega}{ }^{2}$. Given the target false alarm probability $\overline{P_{f}}$, the threshold $\lambda$ can be determined as

$\lambda=Q^{-1}\left(\overline{P_{f}}\right) \times \sqrt{2 N \sigma_{\omega}^{4}}+N \sigma_{\omega}^{2}$,

Where $Q^{-1}($ ) denotes the inverse Gaussian tail probability $Q$-function. Assuming that the noise uncertainty in the wireless network environment is defined as [1/ $\sigma \sigma \omega 2, \rho \sigma \omega 2]$, where $\rho$ is a constant parameter that computes the size of the uncertainty and $\rho>1$. In the adaptive threshold decision scheme, we have chosen two thresholds $\lambda_{A I} \& \lambda_{A 2}$, the value of maximum noise variance shows the value of upper threshold $\lambda_{A l}$ and the value of minimum noise variance shows the value of lower threshold $\lambda_{A 2}$. Hence

$\lambda_{A 1}=Q^{-1}\left(\overline{P_{f}}\right) \times \sqrt{2 N \rho \sigma_{\omega}^{4}}+N \rho \sigma_{\omega}^{2}$

$\lambda_{A 2}=Q^{-1}\left(\overline{P_{f}}\right) \times \sqrt{2 N /\left(\rho \sigma_{\omega}^{4}\right)}+N /\left(\rho \sigma_{\omega}^{2}\right)$,

If detected signals fall inside any one of the quantized interval, it will generate its respective decimal values $(D V)$ as

$D V=\left\{\begin{array}{l}\text { If } M=00, \text { respective decimal value }-0 \\ \text { If } M=01, \text { respective decimal value }-1 \\ \text { If } M=10, \text { respective decimal value }-2 \\ \text { If } M=11, \text { respective decimal value }-3\end{array}\right.$

Equation (16) shows the decimal values $(D V)$, which are compared with thresholds $\left(\lambda_{A 1}\right.$, $\left.\lambda_{A 2}, \& \lambda_{2}\right)$ to make local decision at a fixed $P_{f}$ i.e. 0.1. Outside the confused region it generates binary bit i.e. 0 or 1 depends on signal existence.

\section{Proposed System Model}

\section{A. Proposed ESNR_ADT Spectrum Sensing Scheme}

Figure 3, shows proposed ESNR_ADT spectrum sensing scheme. CR receiver receives PU signal and calculates SNR value $\left(S_{e}\right)$ using above mentioned mathematical formulas. Now, compare the value of $\left(S_{e}\right)$ with decided threshold $(\gamma)$ in order to select detector for $\mathrm{PU}$ signal detection. If the value of estimated SNR is greater or equal to threshold then ED will use. Otherwise, ED with ADT will detect PU signal.

In Figure 3, assuming $S_{e}$ is greater or equal to $(\gamma)$ then ED is selected and calculates the energy of PU signal $(X)$, compares $(X)$ with threshold $\left(\lambda_{1}\right)$ to indicate $\mathrm{PU}$ is present or absent. At other end if $S_{e}$ is smaller than $(\gamma)$ then ED with ADT is selected and ED with ADT calculates the energy of received PU signal $(Z)$, compares $(Z)$ with thresholds $\left(\lambda_{A I}\right.$ $\& \lambda_{A 2}$ ) by using adaptive threshold scheme. Finally, comparethe output value of ED with ADT i.e. $(Y)$ to threshold $\left(\lambda_{2}\right)$ under considering a fixed $P_{f}$ i.e. 0.1 , to determine whether PU 
is present or not. Propose model chooses one detector between ED and ED with ADT with the help of mentioned mathematical expression given as

Selection of detector $= \begin{cases}E D, & S_{e} \geq \gamma \\ E D \_A D T, & S_{e}<\gamma\end{cases}$

The probability of detection $P_{d}$ can be also identified. The expression for $P_{f}$ and $P_{d}$ can be defined as [14].

$$
\begin{aligned}
& P_{f}=P_{r}(X<\lambda)=Q\left(\frac{\lambda-N \sigma_{\omega}^{2}}{\sqrt{2 N \sigma_{\omega}^{4}}}\right), \\
& P_{d}=P_{r}(X \geq \lambda)=Q\left(\frac{\lambda-N\left(\sigma_{S}^{2}+\sigma_{\omega}^{2}\right)}{\sqrt{2 N\left(\sigma_{S}^{2}+\sigma_{\omega}^{2}\right)^{2}}}\right),
\end{aligned}
$$

Where, $\sigma_{\omega}{ }^{2}$ and $\sigma_{S}{ }^{2}$ are the noise variance and signal variance respectively. $Q($ ) denotes Gaussian tail probability $Q$-function. The total error rate is the sum of the probability of false alarm $(P f)$ and the probability of missed detection alarm $(P m)$. Hence, the total error probability rate as follows

$P_{e}=P_{f}+P_{d}$

$P_{e}=P_{f}+\left(1-P_{d}\right)$,

Where, $\left(1-P_{d}\right)$ shows the probability of missed detection $\left(P_{m}\right)$.

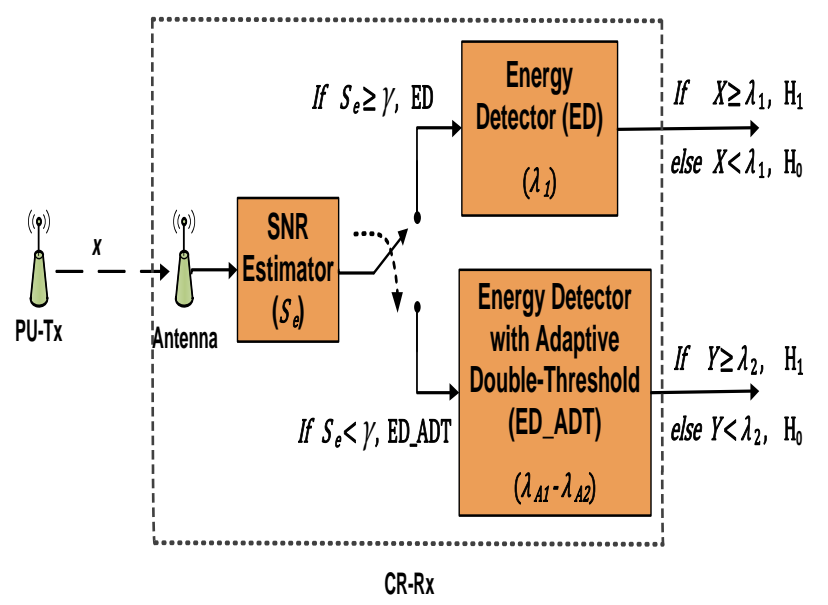

Figure 3. Proposed Model: SNR Estimation Based Spectrum Sensing Detector (ESNR_ADT)

\section{A.1. Energy Detector with Single Threshold (ED)}

Figure 4, shows the internal architecture of ED [15] with single threshold $\left(\lambda_{1}\right)$. Here, input PU licensed signal received by square law device, which shows detected signal 
energy $(X)[16]$ and compared with single threshold to make a final decision to determine whether the PU is present or absent.

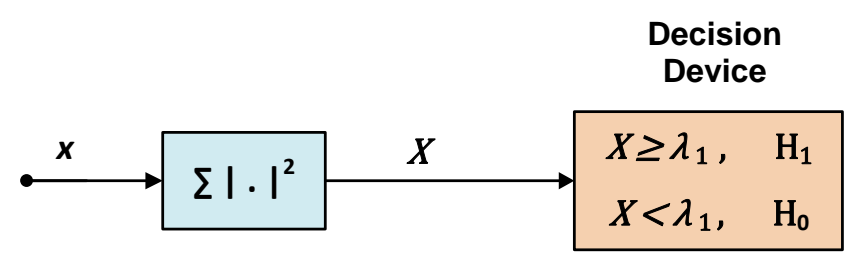

Figure 4. Internal Architecture of Energy Detector with Single Threshold (ED)

$X=\frac{1}{N} \sum_{n=1}^{N}|x(n)|^{2}$,

First stage local decision rule $(L F)$ used by energy detector with single threshold is given by

$L F= \begin{cases}1, & \lambda_{1} \leq X \\ 0, & X<\lambda_{1}\end{cases}$

\section{A.2. Energy Detector with AdaptiveTthreshold (ED with ADT)}

This is a simple ED circuit except threshold, where we used adaptive threshold instead of fixed threshold. Suppose that estimated SNR value is lesser than decided threshold $(\gamma)$ then ED with ADTdetector will try to detect PU signal. Figure 5 shows model of ED with ADT where firstly, square law device (SLD) detects the signal and shows signal energy (Z). After SLD, we have two parts named as upper part and lower part. In upper part if detected energy values $(Z)$ are greater than or equal to $\lambda_{A l}$, it will show $H_{l}$ (signal presented), or less than $\lambda_{A 2}$ show $H_{0}$ (signal absent). But, if detected energy values $(Z)$ fall between $\lambda_{A 1}$ and $\lambda_{A 2}$ then it will consider lower part and follow quantization process to produce its respective decimal values (DV) as shown in equation (16) under the consideration that $P_{f}$ should be 0.1 .

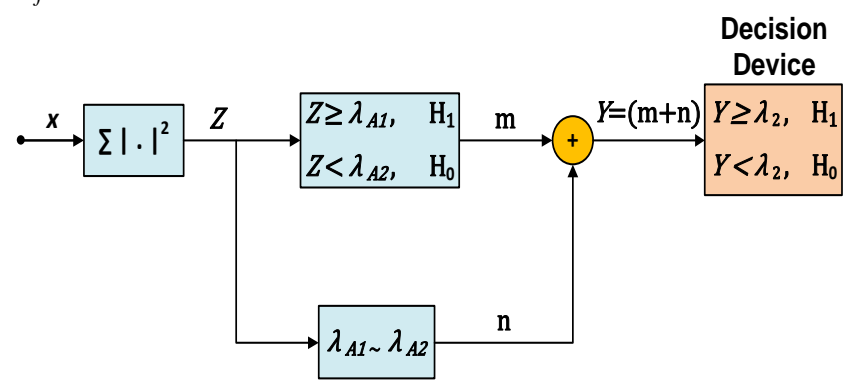

Figure 5. Internal Architecture of Energy Detector with Adaptive Threshold (ED with ADT)

If detected energy values $(Z)$ fall outside or between $\lambda_{A l}$ and $\lambda_{A 2}$, using Equation $(8,11 \&$ 16) it generates value as

$$
\begin{aligned}
& m=\left\{\begin{array}{lc}
0, & Z<\lambda_{A 2} \\
1, & \lambda_{A 1} \leq Z
\end{array}\right. \\
& n=\left\{D V, \quad \lambda_{A 2}<X<\lambda_{A 1}\right.
\end{aligned}
$$


Where, $m$ and $n$ are the output values of upper part and lower part respectively. After that values of $m$ and $n$ are added using adder.

$Y=(m+n)$

Finally, Second stage local decision $(L S)$ is expressed using equation (24), (25) \& (26), which is the final output of ED with ADT as follows:

$$
L S= \begin{cases}1, & \lambda_{2} \leq Y \\ 0, & Y<\lambda_{2}\end{cases}
$$

Equation (27), comparing the resultant value $(Y)$ to threshold $\left(\lambda_{2}\right)$, to maintainoverall system probability of false alarm $\left(P_{f}\right) 0.1$. If $Y$ is greater than $\lambda_{2}$ signal is present otherwise absent.

The novelty of this paper that there are two detectors but implies single stage detector scheme. Based on estimated SNR value,proposed model will chose respective detector. This propose scheme improves sensing time, detection performance, overcome sensing failure problem, and reduce computational complexity as well.

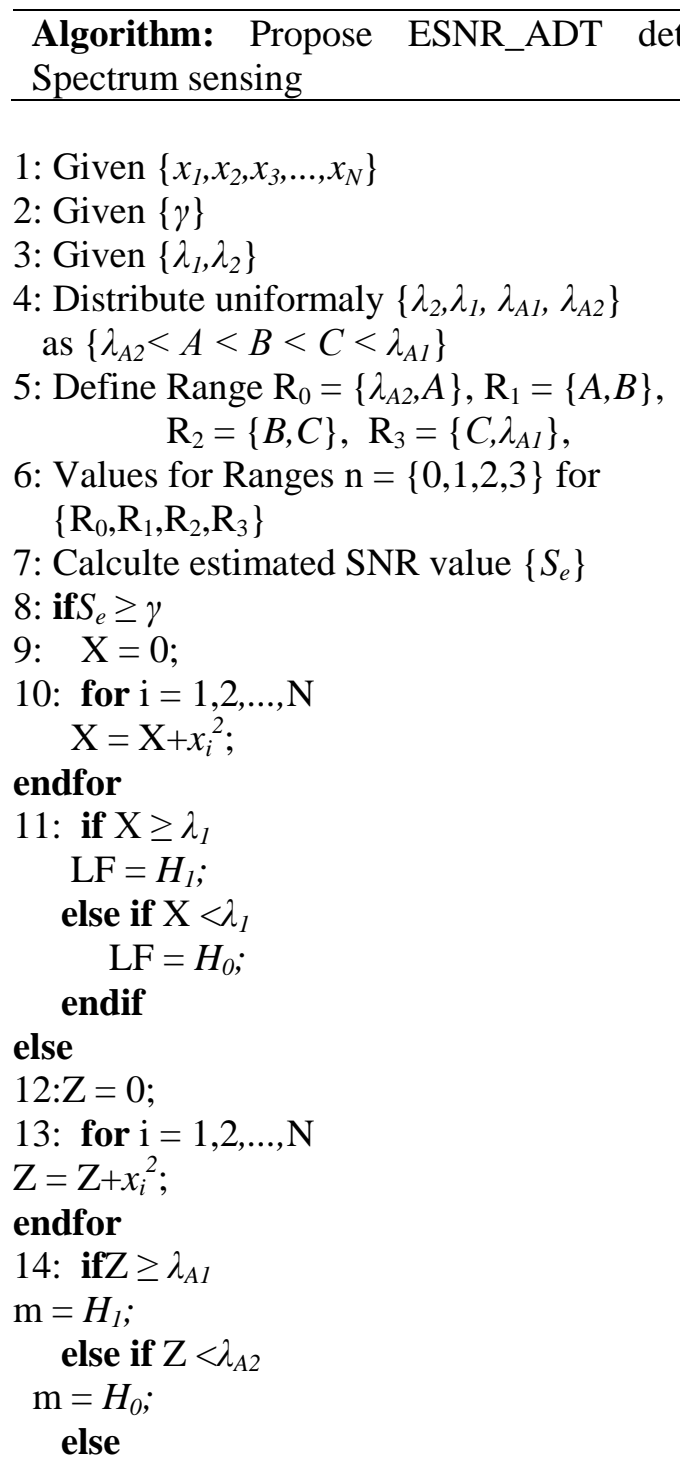



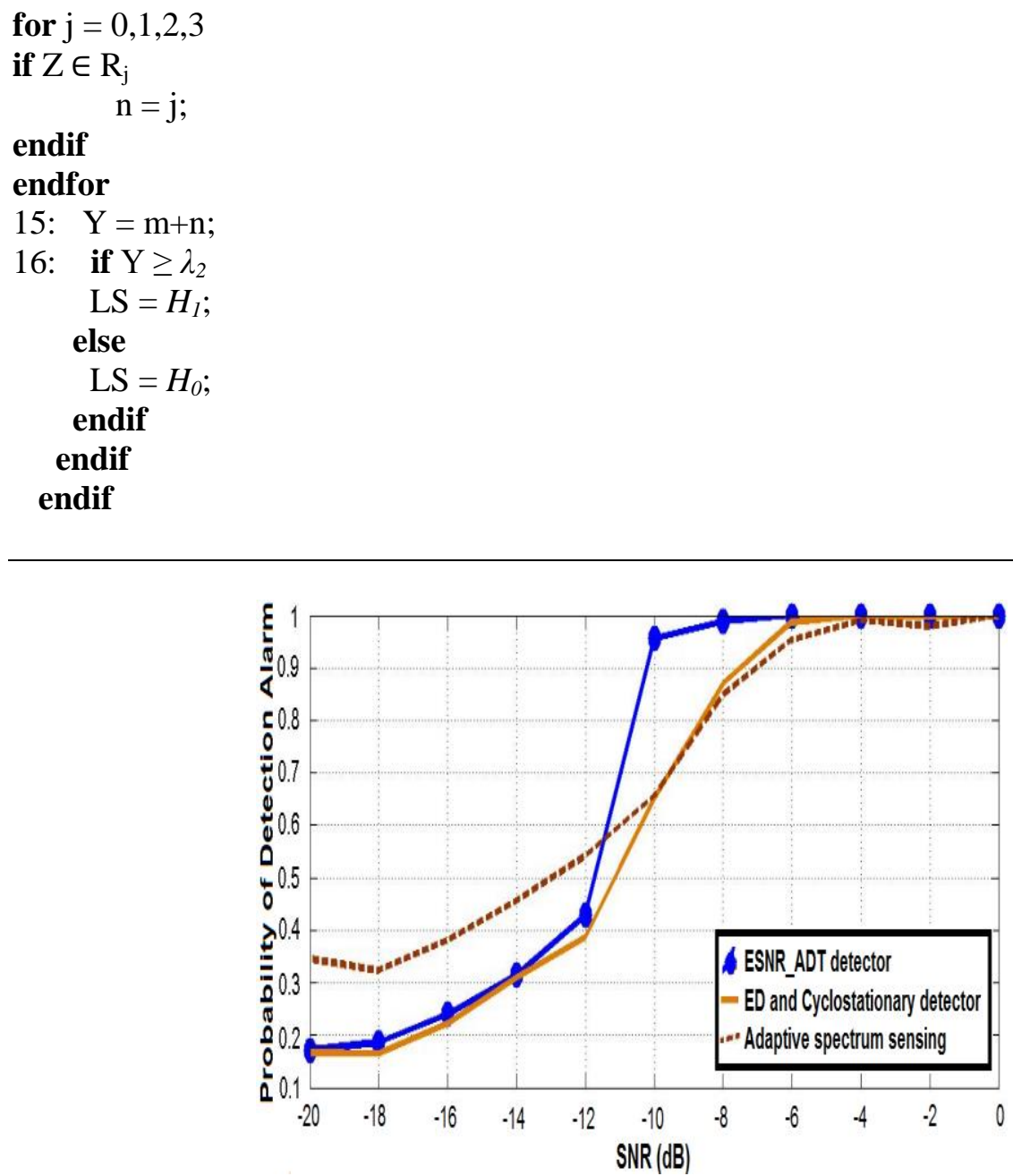

Figure 6. Probability of Detection Vs SNR at $P_{f}=0.1$ with $N=1000$, QPSK Modulation Scheme and Rayleigh Fading Channel

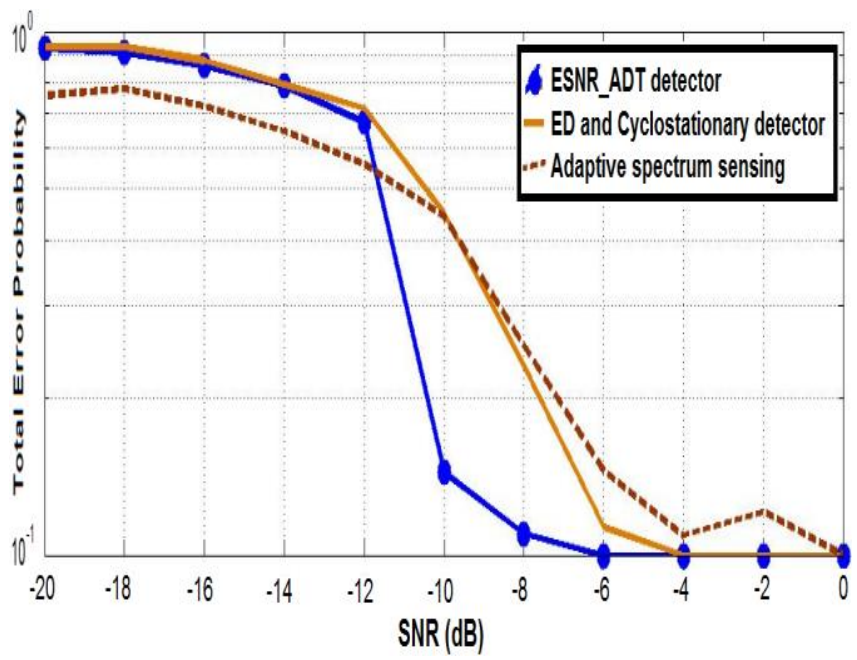

Figure 7. Total Error Probability Vs SNR at $P_{f}=0.1$ with $N=1000$, QPSK Modulation Scheme and Rayleigh Fading Channel 


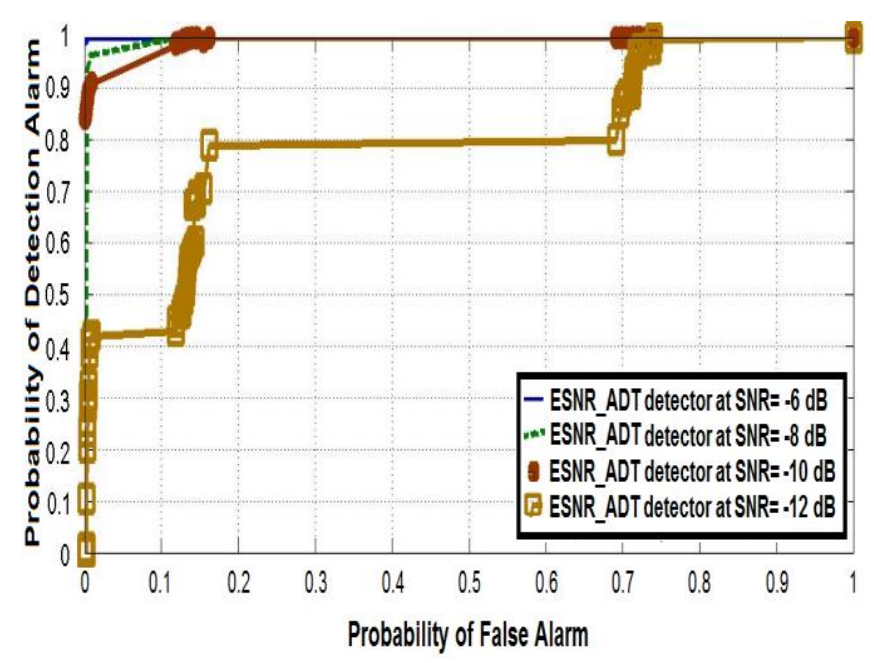

Figure 8. ROC Curves for ESNR_ADT Based Spectrum Sensing Detector under Different SNR Values

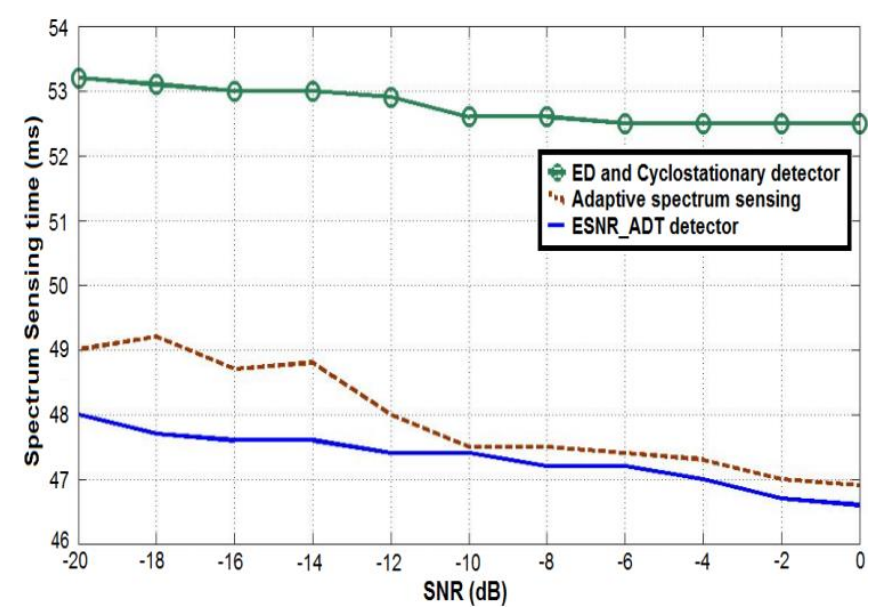

Figure 9. Spectrum Sensing Time Vs SNR withN= 1000, QPSK Modulation Scheme and Rayleigh Fading Channel

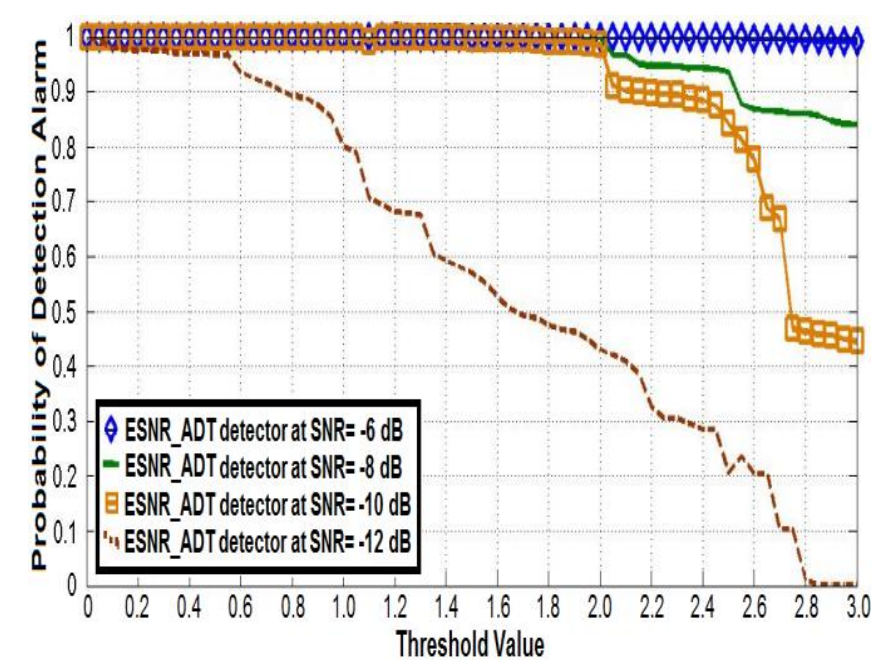

Figure 10. Probability of Detection Vs Threshold Values at SNR =-6 dB, -8 dB, $-10 \mathrm{~dB},-12 \mathrm{~dB}$, with $N=1000$, QPSK Modulation Scheme and Rayleigh Fading Channel 


\section{Numerical Results And Analysis}

In the presented system model, we assumed total number of samples $(N) 1000, P_{f}=0.1$, and SNR varies from - $20 \mathrm{~dB}$ to $0 \mathrm{~dB}$. QPSK modulation is considered in Rayleigh fading channel.

The probability of detection describes how often PU is susceptible to potential interference from the CR. In CRN, $P d$ is one of the important parameter in order to calculate the performance of system. According to IEEE 802.22 WRAN standard the value of $P d$ should be as maximum as possible under the constraint of probability of false alarm.

Figure 6 exhibits the graph between $P_{d}$ and SNR of the proposed scheme with other two previous schemes. It is found that our scheme yields better results and the detection performance is improved by $30.5 \%$ and $30 \%$ as compare to cyclostationary based sensing method and adaptive SS at $-10 \mathrm{~dB}$ SNR respectively.

Figure 7 shows that the proposed scheme has minimum error rate as compared to other two previous schemes, i.e. 0.1 at $-6 \mathrm{~dB}$ SNR.

Receiver Operating Characteristics (ROC) curve is illustrates in Figure 8. ROC curve shows the relationship between probability of false alarm and probability of detection alarm [17] of a SS method under several SNR values for propose scheme. For $P_{f}=0.1$ and $\mathrm{SNR}=-10 \mathrm{~dB}$, probability of detection is in the order of 0.9 , which is the SS requirement of IEEE 802.22 [18-19].

The SS time defines the total time taken by CR user to detect PU signal. Suppose SS time is increased then PU can utilize its spectrum in a better manner and the limit is decided that CR can't interfere throughout that much of time. More PUs will be detected if more the SS, due to this the level of interference will be less. The SS time is directly related to the number of samples received by the CR user. The more sensing time is devoted to detecting, the less sensing time is available for transmissions and hence degrading the CR throughput. This is known as the sensing efficiency problem [20] or the sensing-throughput tradeoff [21] in SS.

Figure 9 shows the graph of spectrum sensing time versus SNR. The proposed scheme requires lesser sensing time than the previously proposed schemes. It is observed that there is an inverse relation between SS time and SNR. As SNR increases, sensing time decreases. At - $20 \mathrm{~dB}$ SNR, proposed scheme requires approximately $48 \mathrm{~ms}$ while previous schemes (cyclostationary based sensing method and adaptive SS) requires around $53.2 \mathrm{~ms}$ and $49.0 \mathrm{~ms}$ sensing time.

$T=T_{F}+T_{S}$

In Equation (28), Tshows total SS time of CR user. $T_{F}$ and $T_{S}$ are the first stage SS time and second stage SS time of individual CR user's respectively.

In Figure 10, we have plotted the probability of detection $\left(P_{d}\right)$ versus Threshold value ( $\lambda$ ) plots for different SNR values such as $-6 \mathrm{~dB},-8 \mathrm{~dB},-10 \mathrm{~dB}, \&-12 \mathrm{~dB}$, it is seen that there is an inverse relationship between $\left(P_{d}\right)$ and threshold for a fixed value of SNR. Observe Figure 10, if SNR increases, probability of detection also increases with respect to threshold. The maximum value of probability of detection is approximately 1.0 throughout the range of threshold $(\lambda)$ at $-6 \mathrm{~dB}$ SNR. It shows that the proposed ESNR_ADT detector can detect PU signal at $-6 \mathrm{~dB}$ SNR for $N=1000$, and $\lambda=3.0$.

\section{Conclusion}

In this paper, we have proposed a robust detector using SNR with adaptive threshold scheme in CRN. This scheme improves sensing time and overcomes sensing failure problems. Numerical results show that proposed ESNR_ADT scheme outperforms other two previous schemes, by $30 \%$ and $30.5 \%$ at $-10 \mathrm{~dB}$ SNR. It is also shown that the proposed scheme yields lesser sensing time than cyclostationary detection and adaptive SS 
scheme, by $5.2 \mathrm{~ms}$ and $1.0 \mathrm{~ms}$ at $-20 \mathrm{~dB}$ SNR respectively. All results conclude that the proposed scheme exhibits better performances than previous schemes.

\section{References}

[1] A. Bagwari and B. Singh, "Comparative performance evaluation of Spectrum Sensing Techniques for Cognitive Radio Networks," Fourth IEEE International Conference on Computational Intelligence and Communication Networks (CICN- 2012), vol. 1, (2012), pp. 98-105.

[2] A. Bagwari and G. S. Tomar, "Comparison between Adaptive Double-Threshold Based Energy Detection and Cyclostationary detection technique for Cognitive Radio Networks," Fourth IEEE International Conference on Computational Intelligence and Communication Networks (CICN-2013), vol. 1, (2013), pp. 182-185.

[3] L. Lu, X. Zhou, U. Onunkwo and G. Y. Li, "Ten years of research in spectrum sensing and sharing in cognitive radio," In EURASIP Journal on Wireless Communications and Networking, (2012) January, pp. 1-16.

[4] A. Bagwari and G. S. Tomar, "Adaptive Double-Threshold Based Energy Detector for Spectrum Sensing in Cognitive Radio Networks", International Journal of Electronics Letters (IJEL) - Taylor \& Francis Group, pp 33-41, (2013) April 04.

[5] Y. Zeng, Y.-C. Liang, A. T. Hoang and R. Zhang, "A Review on Spectrum Sensing for Cognitive Radio: Challenges and Solutions", EURASIP Journal on Advances in Signal Processing, vol. 2010, (2010), pp. $1-15$.

[6] S.Maleki, A. Pandharipande and G. Leus, "Two-Stage Spectrum sensing for cognitive radios," IEEE conference on Acoustics Speech and Signal Processing (ICASSP), (2010), pp. 2946-2949.

[7] W. Ejaz, N. U. Hasan and Hyung Seok Kim, "SNR-BASED ADAPTIVE SPECTRUM SENSING FOR COGNITIVE RADIO NETWORKS," International Journal of Innovative Computing, Information and Control, vol. 8, no. 9, (2012) September, pp. 6095-6105.

[8] J. Vartiainen, H. Saarnisaari, J. J. Lehtomaki and M. Juntti, "A blind signal localization and SNR estimation method", Proc. of IEEE Military Communication Conference, pp.1-7, (2006).

[9] S.-Q. Liu, B.-J. H and X.-Y. Wang, "Hierarchical Cooperative Spectrum Sensing Based on Double Thresholds Energy Detection," IEEE Communications Letters, vol. 16, no. 7, (2012) July, pp. 10961099.

[10] A. Ghasemi and E. S. Sousa, "Collaborative spectrum sensing for opportunistic access in fading environments," in Proc. IEEE International symposium on New Frontiers in Dynamics Spectrum Access Networks, pp. 131-136, (2005) November 8-11.

[11] L.-L. ZHANG, J. G. HUANG and C. K. TANG, "Novel Energy Detection Scheme in Cognitive Radio," IEEE conference on Signal Processing, Communications and Computing (ICSPCC), (2011), pp. 1-4.

[12] R. Tandra and A. Sahai, "SNR Walls for Signal Detection," IEEE Jour. of Slected Topic in Sig. Proc., vol. 2, no. 1, (2008) Febuary, pp. 4-16.

[13] C. H. Sun, W. Zhang and K. Ben Letaief, "Cooperative spectrum sensing for cognitive radios under bandwidth constraints," in Proc. 2007 IEEE Wireless Communications and Networking Conference, vol. 1-9, (2007), pp. 1-5.

[14] M. L.-Benítez and F. Casadevall, "Improved Energy Detection Spectrum Sensing for Cognitive Radio," In IET Communications, vol. 6, no. 8, (2012) July 09, pp. 785-796.

[15] H. Urkowitz, "Energy detection of unknown deterministic signals," In Proc. IEEE, vol. 55, no. 4, pp. 523-531, Apr. 1967

[16] D. Chen, J. Li and J. Ma, "Cooperative Spectrum Sensing under Noise Uncertainty in Cognitive Radio," Wireless Communications, Networking and Mobile Computing, 2008, ISBN: 978-1-4244-2108-4, (2008), pp. 1-4.

[17] T. Yucek and H. Arslan, "A Survey of Spectrum Sensing Algorithms for Cognitive Radio Applications," IEEE Communication Serveys and Toutorials, , First Quarter, vol. 11, no. 1, (2009), pp. 116-130.

[18] T. Do and B. L. Mark, "Improving Spectrum Sensing Performance by Exploiting Multiuser Diversity," Foundation of Cognitive Radio Systems, Prof. Samuel Cheng (Ed.), ISBN: 978-953-51-0268-7, (2012) March, pp. 119-140.

[19] C. Cordeiro, K. Challapali, D. Birru and S. Shankar,: "The first worldwide wireless standard based on cognitive radios", in Proceedings of DySPAN 2005, IEEE, vol. 802, no. 22, (2005) November.

[20] W. Y. Lee and I. F. Akyildiz, "Optimal spectrum sensing framework for cognitive radio networks," IEEE Transactions on Wireless Communications, vol. 7, no.10, (2008), pp. 3845-3857.

[21] Y. C. Liang, Y. Zeng, E. Peh and A.T. Hoang, "Sensing-throughput tradeoff for cognitive radio networks," IEEE Transactions on Wireless Communications, vol. 7, no. 4, (2008), pp. 1326-1337.

[22] J. Kanti and G. S. Tomar, "Various Sensing Techniques in Cognitive Radio Networks: A Review", International Journal of Grid and Distributed Computing, vol. 9, no. 1, (2016), pp.145-154.

[23] A. Bagwari and G. S. Tomar, "Multiple Energy detectors Based Cognitive Radio Networks Using Adaptive Double-Threshold scheme", IJSBT, vol. 1, no.1, (2013), pp.17-27. 
[24] A. Bagwari and G. S. Tomar, "Performance Study between Two-Stage Detectors and Estimated SNR Based Detector in ognitive Radio Networks", IEEE International Conference on Computational Intelligence and Communication Networks, (2014), pp.425-428.

[25] A. Bagwari and G. S. Tomar, "Enriched the Spectrum Sensing Performance of Estimated SNR Based Detector in Cognitive Radio Networks", International Journal of Hybrid Information Technology, vol.8, no.9, (2015), pp.143-156,. 
International Journal of Signal Processing, Image Processing and Pattern Recognition Vol. 9, No. 5 (2016) 